Julius Scharnetzky

Flossenbürg Memorial, Germany

\title{
Die KZ-Gedenkstätte Flossenbürg - ein Ort des Gedenkens, der Begegnung und des historischen Lernens
}

\section{Historischer Überblick zur Lagergeschichte}

Der deutsche Essayist Max Goldt bezeichnete das Konzentrationslager

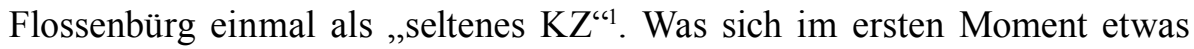
sonderbar anhören mag, ist im Kern aber nicht verkehrt, denn bis heute ist das einstige Konzentrationslager vielen in seiner Bedeutung nicht bekannt. Während Lager wie Auschwitz, Buchenwald oder Dachau seit vielen Jahren, gar Jahrzehnten, Metaphern für die nationalsozialistische Verfolgungs- und Vernichtungspolitik und das Leid von Millionen Menschen sind, wird das Konzentrationslager Flossenbürg im historischen und erinnerungspoltischen Diskurs lange Zeit nur als Randnotiz wahrgenommen.

Als das KZ Flossenbürg im Frühjahr 1938 gegründet wird, dienen die Konzentrationslager schon lange nicht mehr einzig der Unterdrückung politischer Gegner. Vielmehr werden ab 1937/1938 zehntausende Menschen verhaftet und als Maßnahme präventiver Verbrechensbekämpfung in die Konzentrationslagerüberstellt, die gemäß dernationalsozialistischen Ideologie als sogenannte „Berufsverbrecher“ beziehungsweise als „Asoziale“ gelten. Die Lager als Haftorte für angebliche „Volksgegner“ unterschiedlicher Couleur, scheinen, nicht zuletzt aufgrund der Propaganda der vorangegangenen Jahre, von der Gesellschaft bereits weitegehend als systemimmanent hingenommen worden zu sein.

1 Vgl. M. Goldt, Ich beeindrucke durch ein seltenes KZ, in: M. Goldt, Quitten für die Menschen zwischen Zittau und Emden, Zürich 1993, S. 204-208. 
Unter menschenunwürdigen Bedingungen beutet die SS die Arbeitskraft der Häftlinge vor allem bei der Produktion von Baustoffen aus. Zu diesem Zweck gründet sie gar einen eigenen Wirtschaftsbetrieb - die Deutschen Erd- und Steinwerke, kurz DESt. Die erzielten Gewinne bleiben aber weit hinter den wirtschaftlichen Erwartungen der SS zurück. Die unausgebildeten, schlecht versorgten Häftlinge, die überdies permanenter Gewalt ausgesetzt sind und tagtäglich um ihr Leben fürchten müssen, sind überhaupt nicht in der Lage, Werksteine in der Qualität und Quantität zu produzieren, wie diese für die monumentalen Bauprojekte in Berlin, Nürnberg und den anderen ,Führerstädten“ benötigt werden. Stattdessen fertigen sie hauptsächlich Straßenbaumaterial. Die Steinbrüche sind daneben aber auch Orte, an denen die Häftlinge drangsaliert werden und häufig sinnlose Arbeiten verrichten müssen².

Die Granitvorkommen in Flossenbürg sind es, die Vertreter der SS Anfang 1938 auf den kleinen verschlafenen Ort in der oberpfälzer Provinz nahe der Grenze zur Tschechoslowakischen Republik aufmerksam werden lassen. Wenige Wochen später treffen am 3. Mai die ersten 100 Häftlinge ein, Ende des Jahres sind bereits 1.500 Männer im Konzentrationslager inhaftiert. Bis zum Ende des Krieges werden etwa 100.000 Menschen, Männer, Frauen und Kinder, aus ganz Europa als Häftlinge im Konzentrationslager Flossenbürg oder einem der 92 Außenlager registriert. 30.000 von ihnen erleben das Kriegsende nicht - sie verhungern, brechen erschöpft zusammen, verletzten sich tödlich bei der Arbeit, werden von Krankheiten dahingerafft oder hingerichtet. Der Großteil der inhaftierten Menschen kommt aus den von Deutschland besetzten Ländern im Osten Europas - über die Hälfte aus Polen (31.400) und der damaligen Sowjetunion (22.000)

$\mathrm{Ab} 1943$ verliert die Produktion der Baustoffe zugunsten der Rüstungsindustrie zusehends an Bedeutung. Zur Deckung des enormen Bedarfs an Arbeitskräften werden verstärkt KZ-Häftlinge herangezogen. Während die Firmen zunächst nur in den Hauptlagern produzieren lassen dürfen, da die SS sonst fürchten muss, die Häftlinge als Machtfaktor zu

2 Vgl. H. Kaienburg, Zwangsarbeit: KZ und Wirtschaft im Zweiten Weltkrieg, in: W. Benz/B. Distel (Hrsg.), Der Ort des Terrors. Geschichte der nationalsozialistischen Konzentrationslager. Die Organisation des Terrors, Bd. 1, München 2005, S. 179-194, hier S. 181f.

3 Zur Geschichte des Lagerkomplexes Flossenbürg siehe ausführlich J. Skriebeleit, Flossenbürg - Stammlager, in: W. Benz/B. Distel (Hrsg.): Der Ort des Terrors. Geschichte der nationalsozialistischen Konzentrationslager. Flossenbürg, Mauthausen, Ravensbrück, Bd. 4, München 2006, S. 17-66. 
verlieren, muss die SS-Führung diesen Anspruch gegen Ende 1943 fallen lassen. Arbeitskommandos bestehend aus KZ-Häftlingen müssen nun auch fernab der Hauptlager in der Rüstungsindustrie Zwangsarbeit verrichten. Zum Lagerkomplex Flossenbürg zählen insgesamt 92 solcher Außenkommandos. Die Überlebenschancen in diesen unterscheiden sich stark voneinander ${ }^{4}$.

\section{Das Gelände des ehemaligen Konzentrationslagers - Erinnern, pragmatisch Nutzen, Verdrängen}

Bereits wenige Wochen nach der Befreiung des Lagers durch USamerikanische Truppen am 23. April 1945 verlassen die letzten noch im Lager verbliebenen Häftlinge Flossenbürg. Das Gelände des Konzentrationslagers (inklusive Steinbruch) mit sämtlichen Mobilien und Immobilien geht in den Besitz der amerikanischen Besatzer über. Diese nutzen den Bereich des ehemaligen Häftlingslagers ab Sommer 1945 als Kriegsgefangenenlager für SS-Angehörige. Ein Jahr später bringen die Vereinten Nationen nichtjüdische Displaced Persons (DPs) polnischer Herkunft auf dem Gelände unter, die während des Krieges als Zwangsarbeiter nach Österreich verschleppt oder im KZ Mauthausen interniert worden waren ${ }^{5}$.

Formen des Erinnerns an die in Flossenbürg verübten Verbrechen existieren mit Ausnahme des in der Ortsmitte (und damit in einiger Entfernung zum einstigen Lagergelände) angelegten Ehrenfriedhofs, auf dem die nach der Befreiung verstorbenen Häftlinge begraben sind, nicht. Es sind die polnischen DPs, die sich vor dem Hintergrund ihrer eigenen Verfolgung durch das nationalsozialistische Deutschland der Erinnerung annehmen und sich für eine würdige Gedenkform einsetzen. Der Initiative des gegründeten Denkmalskomitees können sich weder die örtliche Gemeinde noch staatliche Stellen entziehen. Die in unmittelbarer Nähe zum einstigen Häftlingsbereich entstehende Gedenklandschaft „Tal des Todes“ ist eine der frühesten ihrer Art

4 Vgl. J. Skriebeleit, Flossenbürg - Stammlager, in: W. Benz/B. Distel (Hrsg.): Der Ort des Terrors. Geschichte der nationalsozialistischen Konzentrationslager. Flossenbürg, Mauthausen, Ravensbrück, Bd. 4, München 2006, S. 17-66.

Vgl. Ch. Schikorra, Übergang und Neuordnung. Sommer 1945-1950, in: KZ-Gedenkstätte Flossenbürg/Stiftung Bayerischer Gedenkstätten (Hrsg.): Was bleibt. Nachwirkungen des Konzentrationslagers Flossenbürg. Katalog zur Dauerausstellung, Göttingen 2011, S. 50-55, hier S. 54. 
und vereint christliche sowie nationale Symbole. Den zentralen Erinnerungsort innerhalb dieser Anlage bildet die neugebaute Kapelle „Jesus im Kerker“‘6.

Das „Tal des Todes“ als Erinnerungsort und die Auflösung des DPCamps im Sommer 1947 ermöglichen es den lokalen Entscheidungsträgern, den Fokus auf vermeintlich dringendere Probleme der Zeit zu legen und die leer stehenden Bereiche des einstigen Lagers für die eigenen Bedürfnisse zu nutzen. So werden in den steinernen Gebäuden Flüchtlinge einquartiert, der Appellplatz wirtschaftlich nachgenutzt und die neugegründete Oberpfälzer Steinindustrie betreibt den Steinbruch weiter. 1958 gibt das Finanzministerium des Freistaates Bayern, in dessen Besitz sich die Liegenschaft seit der Gründung der Bundesrepublik Deutschland befindet, dem fast zehn Jahre dauernden Drängen des Ortes Flossenbürg schließlich nach und gestattet die Errichtung einer Wohnsiedlung auf dem früheren Barackengelände. Ein vollständiger Abriss des Arrestbaus kann 1964 nur mit dem Hinweis auf die Bedeutung des Ortes, an dem am 9. April 1945 der Theologe Dietrich Bonhoeffer hingerichtet wurde, aus Kreisen der evangelischen Kirche verhindert werden. Die vielfältige Nachnutzung tilgt die Spuren des Konzentrationslagers in baulicher Sicht fast vollkommen aus der öffentlichen Wahrnehmung und damit den vermeintlichen Makel der Geschichte, der über dem Ort Flossenbürg zu schweben scheint. Formen des Gedenkens bleiben auf das „Tal des Todes“ und den 1957 parkähnlich angelegten Ehrenfriedhof für die Opfer der Todesmärsche beschränkt ${ }^{7}$. Historische Informationen zur Geschichte des Lagers sucht der Besucher zu dieser Zeit vergeblich ${ }^{8}$.

6 Zur Entstehung der ersten Gedenkanlage und deren symbolische Ausgestaltung siehe ausführlich J. Skriebeleit, Erinnerungsort Flossenbürg. Akteure, Zäsuren, Geschichtsbilder, Göttingen 2009, S. 97-160.

7 Die Opfer der, „Todesmärsche“, die aufgrund des nahenden Kriegsendes häufig nur notdürftig am Wegesrand verscharrt worden waren, erhalten auf Weisung der amerikanischen Besatzer nach der Befreiung ein würdiges Begräbnis. Ihre Gräber sind über ganz Bayern verteilt. Da es sich nicht um die eigenen Toten handelt, verwahrlosen diese Gräber zusehends. Internationaler Protest und beginnende Umbettungen von französischen und belgischen Toten in ihre Heimatländer zwingen die bayerische Landesregierung zum Handeln. Infolgedessen werden in Dachau und Flossenbürg Sammelgräber angelegt.

8 Vgl. A. Schmidt, Museum, Gedenkstätte, Lernort - die KZ-Gedenkstätte Flossenbürg als Bildungsort für Jugendliche, in: B. Schoßig (Hrsg.), Historisch-politische Bildung und Gedenkstättenarbeit als Aufgabe der Jugendarbeit in Bayern. Einrichtungen - Projekte - Konzepte (Dachauer Diskurse. Gautinger Protokolle, Bd. 41), München 2011, S. 197-206, hier S. $198 \mathrm{f}$. 
Während ab Mitte der 1960er Jahre anderenorts in Deutschland große Gedenkstätten entstehen und Gedenkveranstaltungen abgehalten werden, bleibt es in Flossenbürg ruhig. Dieses Engagement ist in der Bundesrepublik Deutschland allerdings weniger auf das Verlangen der Mehrheitsgesellschaft zurückzuführen ${ }^{9}$, die sicherlich immer noch lieber einen Teppich des Schweigens über die eigene Vergangenheit gelegt hätte, als vielmehr auf ehemalige deutsche Häftlinge dieser Orte. Anders als beispielsweise in Dachau fehlen in Flossenbürg jedoch prominente deutsche Häftlinge, die die Memorialisierung hätten einfordern können. Stattdessen werden viele von ihnen aufgrund ihrer einstigen Verhaftungsgründe erneut verfolgt, stigmatisiert oder schlichtweg nicht als Opfer des Nationalsozialismus anerkannt. Darüber hinaus machen es der Systemkonflikt sowie das Nichtsprechendürfen oder -können im sowjetischen Einflussbereich für die ehemaligen Häftlinge, die aus dem Osten Europas stammen, unmöglich den Ort der eigenen Verfolgung aufzusuchen und sich für ein würdiges Gedenken einzusetzen. Dass der Ort dennoch nicht ganz aus dem kollektiven Gedächtnis verschwindet, ist ab Ende der 1950er/Anfang der 1960er Jahre eng mit der Umdeutung Bonhoeffers und anderer Verschwörer des 20. Juli 1944, die in Flossenbürg ermordet worden waren, zu zentralen Akteuren des deutschen Widerstandes verknüpft ${ }^{10}$. Während dieser Männer jährlich gedacht wird, wird die Mehrheit der Häftlinge jedoch beinahe gänzlich aus den Erinnerungshandlungen ausgeschlossen ${ }^{11}$.

9 Der Umgang mit der nationalsozialistischen Vergangenheit und damit auch mit den baulichen Relikten ist in der Deutschen Demokratischen Republik stets politisch motiviert und dient der Parteiführung zur Inszenierung des Antifaschismus als staatstragende Doktrin. Die ehemaligen Lagerorte Buchenwald, Sachsenhausen und Ravensbrück werden zu Nationalen Mahn- und Gedenkorten erhoben. Gleichzeitig bedeutet dies aber auch, dass die Orte und Opfergruppen, die nicht für ideologische Zwecke instrumentalisiert werden können, aus dem Gedenken herausfallen.

${ }^{10}$ Die Attentäter des 20. Juli 1944 gelten bis weit in die 1950er Jahre hinein vielen Zeitgenossen als ,Vaterlandsverräter“.

${ }^{11}$ Nichtsdestotrotz gibt es einzelne Ländergruppen, zum Beispiel die französische Association des Déportés de Flossenbürg et Kommandos, die jährlich nach Flossenbürg kommen, um den Opfern ihrer Länder zu gedenken. Vgl. K. Helldorfer, Verdrängen und Vergessen. 1958-1970, in: KZ-Gedenkstätte Flossenbürg/Stiftung Bayerischer Gedenkstätten (Hrsg.): Was bleibt. Nachwirkungen des Konzentrationslagers Flossenbürg. Katalog zur Dauerausstellung, Göttingen 2011, S. 94-99, hier S. 99; J. Skriebeleit, Selektives Erinnern. 1970-1980, in: KZ-Gedenkstätte Flossenbürg/Stiftung Bayerischer Gedenkstätten (Hrsg.): Was bleibt. Nachwirkungen des Konzentrationslagers Flossenbürg. Katalog zur Dauerausstellung, Göttingen 2011, S. 116-121, hier $120 f$. 


\section{The Person and the Challenges \\ 276 Volume 4 (2014) Number 2, p. 271-280 \\ 3. Die langsame Wiederentdeckung des Erinnerungsortes Flossenbürg}

Die 1980er Jahre bilden in der Bundesrepublik Deutschland eine Zäsur im Umgang mit der nationalsozialistischen Vergangenheit, zunehmend setzen sich lokalgeschichtliche Initiativen kritisch mit der bislang tabuisierten Rolle ihrer Heimatorte im Nationalsozialismus auseinander, fordern die Erhaltung der verbliebenen baulichen Reste und einen angemessenen Umgang. Das wachsende Interesse vor allem der jungen Generation an der Geschichte des Holocaust trägt dazu bei, dass viele Überlebende des NS-Terrors ihr Schweigen beenden und beginnen, ihre Geschichte zu erzählen. Diese Entwicklung geht an Flossenbürg zwar nicht gänzlich vorbei, zum Beispiel entdecken lange vergessene Opfergruppen das Gelände des einstigen $\mathrm{KZ}$ als Gedenkort für sich, doch die bayerische Schlösser- und Seenverwaltung hält weiterhin an der Erhaltung der parkähnlichen Anlage fest und verweist auf die Funktion des Geländes als Friedhof. Als einzige Reaktion auf kritische Äußerungen bezüglich des Zustandes der Anlage stimmt die Schlösser- und Seenverwaltung nach längerer Weigerung einer kleinen Ausstellung über die Lagergeschichte zu, die 1985 im ehemaligen Arrestbau eröffnet wird.

Erst der 50. Jahrestag der Befreiung des Lagers 1995 kann auch in Flossenbürg ein längst überfälliges Umdenken darüber in Gang setzen, wie ein verantwortungsvoller Umgang mit den Überresten des Konzentrationslagers und seiner Geschichte gestaltet werden kann. Vor allem ehemalige Häftlinge äußern sich zunehmend kritisch über die bisherigen Versäumnisse und bauen so gesellschaftlichen Druck auf. Die Zustimmung der Bundesrepublik Deutschland, sich an den Kosten der Gedenkstättenentwicklung zu beteiligen und die Übertragung des Bereiches des einstigen Appellplatzes mit der Häftlingsküche und der Lagerwäscherei, die seit mehr als 50 Jahren industriell genutzt wurden, durch den letzten Eigentümer an den Freistaat Bayern, ermöglichen den Aufbau einer ,arbeitenden Gedenkstätte“, der noch immer andauert.

Als solche ,arbeitenden Gedenkstätte“ versteht sich die KZ-Gedenkstätte Flossenbürg heute. Sie ist ein Ort, an dem geforscht, archiviert, gelernt und über das Gelernte gestritten wird. Zu den Kernaufgaben der KZGedenkstätte Flossenbürg zählt die Betreuung ehemaliger Häftlinge und ihrer Angehörigen. So findet jährlich das dreitägige Treffen der ehemaligen Häftlinge des Konzentrationslagers Flossenbürg und seiner Außenlager statt, an dem neben den ehemaligen Häftlingen und ihren Familien auch andere 
interessierte Personen teilnehmen. Gleichzeitig ist die Gedenkstätte aber auch ein Friedhof für mehr als 5.000 Tote sowie der Ort an dem stellvertretend allen Opfern des Lagerkomplexes gedacht wird. Neben Raum für individuelle Gedenkbekundungen finden auch Gedenkakte von verschiedenen Interessengruppen statt, sodass die Gedenkstätte heute eben nicht mehr ausschließlich ein Friedhof ist, sondern vielmehr ein Erinnerungsort, der der Würde der Opfer angemessen ist und an dem aktiv erinnert wird. Im gedenkstätteninternen Archiv werden zahlreiche zeithistorische Dokumente, Fotografien, etc. gesammelt, die Interessierten - Fachwissenschaftler, Hobbyhistoriker, Schülergruppen, Medienvertreter - zu Forschungszwecken, Schülerprojekten, usw. zur Verfügung gestellt werden. Weiterhin gehören die Bewahrung der historischen Relikte des Konzentrationslager Flossenbürg - Objekte, Bauten, etc. - zur täglichen Arbeit der Gedenkstätte. Nicht zuletzt versteht sich die Gedenkstätte als Lernort, an dem die Geschichte des Nationalsozialismus, des Konzentrationslagers Flossenbürg sowie der Umgang mit der nationalsozialistischen Vergangenheit vermittelt werden. Neben historischen Kommentierungen auf dem ehemaligen Lagergelände und zwei musealen Ausstellungen findet die Vermittlungsarbeit auf der Basis eines breit angelegten Bildungsangebotes statt.

Nach der Fertigstellung des ersten Bauabschnitts im Jahr 2008 ist der ehemalige Appellplatz für die Besucher wieder zugänglich. Außerdem konnte bereits ein Jahr zuvor die erste Dauerausstellung zur Realgeschichte des Lagerkomplexes Flossenbürg in der einstigen Lagerwäscherei eröffnet werden. Die Ausstellung zeichnet sich nicht nur dadurch aus, dass sie gemäß den Standards moderner zeithistorischer Museen konzipiert wurde, sie stellt überdies die Frage nach der zentralen Erzählung der Lagergeschichte: den Menschen. Die Geschichten der Menschen, die im Lagerkomplex Flossenbürg inhaftiert waren, scheinen daher zentral in der Ausstellung auf. Dem Versuch der SS, diese Menschen ihrer Würde und Individualität zu berauben, wirken die Ausstellungsmacher entgegen, in dem sie die ehemaligen Häftlinge nicht nur auf ihren Opferstatus reduzieren, sondern sie als die Individuen präsentieren, die sie waren/sind. 2010 folgte mit „Was bleibt. Nachwirkungen des Konzentrationslagers Flossenbürg“ die zweite Dauerausstellung, die im Gebäude der früheren Häftlingsküche gezeigt wird. In „Was bleibt“ wird ausgestellt, wie mit den Opfern, den Tätern und dem Ort zwischen 1945 und 2010 umgegangen und wie das Erinnern in dieser Zeit immer wieder neu ausgestaltet wird. Ein Jahr nach der Eröffnung erhält die Ausstellung den 
$278 \quad \begin{aligned} & \text { The Person and the Challenges } \\ & \text { Volume 4(2014) Number 2, p. 271-280 }\end{aligned}$

Bayerischen Museumspreis, 2014 eine Special Commendation des European Museum of the Year Awards.

Zurzeit dauern noch die Bauarbeiten für den zweiten Bauabschnitt an, der die Neugestaltung im Außenbereich zur Wiederlesbarmachung des ehemaligen Lagergeländes umfass $\mathrm{t}^{12}$ sowie die Einrichtung eines Bildungshauses im früheren SS-Wirtschaftsgebäude.

\section{Bildungsarbeit in der KZ-Gedenkstätte Flossenbürg}

Die Bildungsarbeit an einem solchen historischen Ort steht vor einem Bündel an Herausforderungen: die Vermittlung historischen Wissens; Wiederlesbarmachung eines stark überformten Geländes; die Arbeit mit den „Bildern im Kopf“ und dem Wissen der Besucherinnen und Besucher über die Zeit des Nationalsozialismus; die Konfrontation mit Gefühlen, die durch die hier verübten Verbrechen ausgelöst werden (können), sowie teilweise normierte Erwartungshaltungen an den Ort eines ehemaligen Konzentrationslagers. Gleichzeitig ist die Einhaltung der Grundlagen der historisch-politischen Bildung, vor allem des Überwältigungsverbots und der Gruppenorientierung, zentral. Daher stellt die KZ-Gedenkstätte ein breit gefächertes methodisches Angebotfürunterschiedliche Zielgruppen und deren Bedürfnisse zur Verfügung. Zentral in den verschiedenen Bildungsangeboten sind lebensgeschichtliche Erzählungen. Die Geschichten und Perspektiven ehemaliger Häftlinge sind in den begleiteten Rundgängen, in der Kleingruppenarbeit in beiden Dauerausstellungen und in Projektarbeiten leitend.

Sämtliche Bildungsangebote werden im Sinne der konstruktivistischen Lerntheorie als aktive Prozesse des gemeinsamen Lernens über die Geschichte des Konzentrationslagers Flossenbürg begriffen, die für jede Gruppe anders sind und die es nie ein zweites Mal geben wird. Subjektorientierung, dialogische Wissensvermittlung, Möglichkeiten zum selbstständigen Entdecken und Forschen, das Verstehen das Geschichtserzählungen nichts Unumstößliches sind, sondern konstruiert werden, Multiperspektivität und die Vermittlung eines möglichst breiten und differenzierten Informationsangebotes sind dabei wesentliche Bestandteile. Der pädagogische Mitarbeiter versteht sich im

${ }^{12}$ Die Außengestaltung muss sich dabei auf die Bereiche beschränken, die heute zur Gedenkstätte gehören und umfasst damit nicht den kompletten SS- bzw. Häftlingsbereich. 
Rahmen dieser Lernprozesse als Moderator, der die Teilnehmer gemäß der sokratischen Mäeutik bei der selbstständigen Konstruktion von Meinungen und Geschichtsbildern unterstützt.

Die Bildungsangebote umfassen ein breites Spektrum an geführten Rundgängen, die an die Bedürfnisse der Gruppen angepasst werden können. Neben den „klassischen Rundgängen“ sind Rundgänge mit thematischen Schwerpunkten in verschiedenen Sprachen buchbar. Des Weiteren bietet die Bildungsabteilung der KZ-Gedenkstätte Flossenbürg einen „Aktivierten Rundgang“ an, der auf dem Konzept „Schüler führen Schüler“ basiert. Anhand von bereitgestellten Materialien erarbeiten sich die Jugendlichen in Kleingruppen ihren eigenen Rundgang. Sie entscheiden dabei nicht nur über die zu vermittelnden Inhalte, sondern auch über die Orte und die Form der Präsentation. Die Wissensvermittlung verliert dabei ihren Primat zugunsten der Einübung unterschiedlicher Kompetenzen und dem Lernen, dass Geschichtserzählungen konstruiert werden. Um den speziellen Bedürfnissen von Jugendlichen mit dem Förderschwerpunkt Lernen zu entsprechen, wurde ein Rundgangsangebot in leichter Sprache entwickelt.

Vertiefende Module, die im Rahmen von halb-, ganz- oder mehrtägigen Seminaren eingesetzt werden können, liegen für beide Ausstellungen vor. Darüber hinaus existieren Konzepte im Bereich der beruflichen Ausbildung der Gesundheitsberufe („Medizin und Konzentrationslager") und der Polizei („Polizei und Konzentrationslager"). Neben dem Erwerb von historischem Wissen setzen sich die Teilnehmer mit konkreten Fragen zum beruflichen Ethos ihrer Berufsgruppe damals und heute auseinander.

Eine weitere zentrale Aufgabe der Bildungsabteilung in Flossenbürg bilden Informations- und Weiterbildungsveranstaltungen für Multiplikatoren im Bereich der schulischen und außerschulischen Bildung.

\section{Perspektiven für die Bildungsarbeit ab 2015}

Anlässlich des 70. Jahrestages der Befreiung des Konzentrationslagers Flossenbürg im April 2015 wird voraussichtlich das Bildungshaus der Gedenkstätte eröffnet werden können, das ein Zentrum für nationales und internationales Lernen über den Holocaust in der Region sein wird. Aufgrund fehlender Seminarräume konnten halb-, ganz- oder mehrtägige Programme bislang nur in sehr eingeschränkter Form durchgeführt werden. Die drei gut 
ausgestatteten Seminarräume des Bildungshauses bieten zukünftig Gruppen einen Raum, sich inhaltlich vertiefter und methodologisch vielfältiger mit der Geschichte des Ortes zu beschäftigen. Neben kognitive Angebote sollen auch kreative treten, die nicht nur einen anderen Zugang ermöglichen, sondern auch andere Formen der Verarbeitung. Ein weiterer Schwerpunktbereich, der momentan im Aufbau begriffen ist, ist die Arbeit mit internationalen Gruppen - zum Beispiel aus der Tschechischen Republik, der Republik Polen und Israel - im Rahmen von Jugendbegegnungen, Austauschprogrammen für Fachkräfte der schulischen und außerschulischen Bildungsarbeit sowie Fachkonferenzen.

Das „seltene Konzentrationslager“, wie es Max Goldt vor über 20 Jahren beschrieben hat, scheint kaum noch zu existieren. Am Ort des einstigen Konzentrationslagers Flossenbürg hat sich durch die Initiative von ehemaligen Häftlingen, interessierten Personen, eines engagierten Teams an Mitarbeitern und nicht zuletzt durch die finanzielle Förderung der Bundesrepublik Deutschland und des Freistaates Bayern vielmehr eine Gedenkstätte entwickelt, die der Würde der Opfer Rechnung trägt und dabei gleichzeitig Räume für historische Arbeit schafft, das Lernen über die Geschichte und die Begegnung. 